\title{
SOME CONNECTIONS BETWEEN AN OPERATOR AND ITS ALUTHGE TRANSFORM
}

\author{
MEE-KYOUNG KIM \\ Department of Mathematics, Sungkyunkwan University, Suwon 440-746, Korea \\ e-mail:mkkim@math.skku.ac.kr \\ and EUNGIL KO* \\ Department of Mathematics, Ewha Women's University, Seoul 120-750, Korea \\ e-mail:eiko@ewha.ac.kr
}

(Received 4 April, 2004; accepted 10 October, 2004)

\begin{abstract}
Associated with $T=U|T|$ (polar decomposition) in $\mathcal{L}(\mathbf{H})$ is a related operator $\tilde{T}=|T|^{\frac{1}{2}} U|T|^{\frac{1}{2}}$, called the Aluthge transform of $T$. In this paper we study some connections between $T$ and $\tilde{T}$, including the following relations; the single valued extension property, an analogue of the single valued extension property on $W^{m}(D, \mathbf{H})$, Dunford's property $(C)$ and the property $(\beta)$.
\end{abstract}

2000 Mathematics Subject Classification. 47B20, 47A15.

Let $\mathbf{H}$ be a complex Hilbert space, and denote by $\mathcal{L}(\mathbf{H})$ the algebra of all bounded linear operators on $\mathbf{H}$. If $T \in \mathcal{L}(\mathbf{H})$, we write $\sigma(T), \sigma_{a p}(T)$, and $\sigma_{p}(T)$ for the spectrum, the approximate point spectrum, and the point spectrum of $T$, respectively.

An arbitrary operator $T \in \mathcal{L}(\mathbf{H})$ has a unique polar decomposition $T=U|T|$, where $|T|=\left(T^{*} T\right)^{\frac{1}{2}}$ and $U$ is the appropriate partial isometry satisfying $\operatorname{ker} U=$ $\operatorname{ker}|T|=\operatorname{ker} T$ and $\operatorname{ker} U^{*}=\operatorname{ker} T^{*}$. Associated with $T$ is a related operator $|T|^{\frac{1}{2}} U|T|^{\frac{1}{2}}$, called the Aluthge transform of $T$, and denoted throughout this paper by $\tilde{T}$.

An operator $T \in \mathcal{L}(\mathbf{H})$ is said to be $p$-hyponormal, where $0<p \leq 1$, if $\left(T^{*} T\right)^{p} \geq$ $\left(T T^{*}\right)^{p}$, where $T^{*}$ is the adjoint of $T$. In particular, if $p=1, T$ is called hyponormal. There is a vast literature concerning $p$-hyponormal operators.

An operator $T \in \mathcal{L}(\mathbf{H})$ is said to satisfy the single-valued extension property if for any open subset $V$ in $\mathbf{C}$, the function

$$
T-\lambda: \mathcal{O}(V, \mathbf{H}) \longrightarrow \mathcal{O}(V, \mathbf{H})
$$

defined by the obvious pointwise multiplication, is one-to-one. Here $\mathcal{O}(V, \mathbf{H})$ denotes the Fréchet space of $\mathbf{H}$-valued analytic functions on $V$ with respect to uniform topology. If $T$ has the single valued extension property, then for any $x \in \mathbf{H}$ there exists a unique maximal open set $\rho_{T}(x)(\supset \rho(T)$, the resolvent set) and a unique $\mathbf{H}$-valued analytic function $f$ defined in $\rho_{T}(x)$ such that

$$
(T-\lambda) f(\lambda)=x \quad\left(\lambda \in \rho_{T}(x)\right) .
$$

\footnotetext{
*The second author is supported by a grant (R14-2003-006-01000-0) from Korea Science and Engineering Foundation.
} 
In the following theorem we show that Aluthge transforms preserve the single valued extension property.

THEOREM 1.1. An operator $T$ with polar decomposition $U|T|$ has the single valued extension property if and only if $\tilde{T}$ has.

Proof. Assume that $T$ has the single valued extension property. Suppose that $W$ is an open subset of $\mathbf{C}$ and $f: W \rightarrow \mathbf{H}$ is an analytic function satisfying $(\tilde{T}-\lambda) f(\lambda)=0$, for each $\lambda \in W$. Since $T\left(U|T|^{\frac{1}{2}}\right)=\left(U|T|^{\frac{1}{2}}\right) \tilde{T}$,

$$
(T-\lambda) U|T|^{\frac{1}{2}} f(\lambda)=U|T|^{\frac{1}{2}}(\tilde{T}-\lambda) f(\lambda)=0,
$$

for each $\lambda \in W$. Since $T$ has the single valued extension property, $U|T|^{\frac{1}{2}} f(\lambda)=0$ for each $\lambda \in W$. Since $\tilde{T}=|T|^{\frac{1}{2}} U|T|^{\frac{1}{2}}, \tilde{T} f(\lambda)=0$ for each $\lambda \in W$. Since $(\tilde{T}-\lambda) f(\lambda)=0$ for each $\lambda \in W, \lambda f(\lambda)=0$ for each $\lambda \in W$. Since $f(\lambda)=0$ on $W \backslash\{0\}$ and is analytic on $W, f$ is identically 0 on $W$. Therefore, $\tilde{T}$ has the single valued extension property.

The proof of the converse implication is similar.

The following corollary shows the relationships between the local spectra of $T$ and $\tilde{T}$.

COROLlARY 1.2. If an operator $T$ with polar decomposition $U|T|$ has the single valued extension property, then

$$
\sigma_{\tilde{T}}\left(|T|^{\frac{1}{2}} x\right) \subset \sigma_{T}(x) \text { and } \sigma_{T}\left(U|T|^{\frac{1}{2}} x\right) \subset \sigma_{\tilde{T}}(x) .
$$

Proof. For $\lambda \in \rho_{T}(x)$, we have $(T-\lambda) x(\lambda) \equiv x$, where $\lambda \rightarrow x(\lambda)$ is the analytic function defined on $\rho_{T}(x)$. Since $|T|^{\frac{1}{2}} T=\tilde{T}|T|^{\frac{1}{2}}$,

$$
(\tilde{T}-\lambda)|T|^{\frac{1}{2}} x(\lambda)=|T|^{\frac{1}{2}}(T-\lambda) x(\lambda) \equiv|T|^{\frac{1}{2}} x .
$$

Hence $\rho_{T}(x) \subset \rho_{\tilde{T}}\left(|T|^{\frac{1}{2}} x\right)$, so that $\sigma_{\tilde{T}}\left(|T|^{\frac{1}{2}} x\right) \subset \sigma_{T}(x)$.

Similarly, we can prove the second inclusion.

COROLlARY 1.3. If an operator $T$ with polar decomposition $U|T|$ has the single valued extension property, then

$$
|T|^{\frac{1}{2}} H_{T}(F) \subseteq H_{\tilde{T}}(F) \text { and } \quad U|T|^{\frac{1}{2}} H_{\tilde{T}}(F) \subseteq H_{T}(F),
$$

where $H_{T}(F)=\left\{x \in \mathbf{H}: \sigma_{T}(x) \subseteq F\right\}$ for $F \subset \mathbf{C}$.

Proof. If $x \in H_{T}(F)$, then $\sigma_{T}(x) \subseteq F$. By Corollary 1.2, we get $\sigma_{\tilde{T}}\left(|T|^{\frac{1}{2}} x\right) \subseteq F$. Hence $|T|^{\frac{1}{2}} x \in H_{\tilde{T}}(F)$. Thus $|T|^{\frac{1}{2}} H_{T}(F) \subseteq H_{\tilde{T}}(F)$.

Similarly, we get $U|T|^{\frac{1}{2}} H_{\tilde{T}}(F) \subseteq H_{T}(F)$.

Our next result shows that the Aluthge transform preserves an analogue of the single valued extension property for $W^{m}(D, \mathbf{H})$ and an operator $T$ on $\mathbf{H}$; that is, $T-\lambda: W^{m}(D, \mathbf{H}) \rightarrow W^{m}(D, \mathbf{H})$ is one-to-one if and only if $\tilde{T}-\lambda$ is. First of all, let us define a special Sobolev type space. Let $D$ be a bounded open subset of $\mathbf{C}$ and $m$ a fixed non-negative integer. The vector valued Sobolev space $W^{m}(D, \mathbf{H})$ with respect to $\bar{\partial}$ and order $m$ will be the space of those functions $f \in L^{2}(D, \mathbf{H})$ whose derivatives $\bar{\partial} f, \cdots, \bar{\partial}^{m} f$ in the sense of distributions still belong to $L^{2}(D, \mathbf{H})$. 
Endowed with the norm

$$
\|f\|_{W^{m}}^{2}=\sum_{i=0}^{m}\left\|\bar{\partial}^{m} f\right\|_{2, D}^{2},
$$

$W^{m}(D, \mathbf{H})$ becomes a Hilbert space contained continuously in $L^{2}(D, \mathbf{H})$.

THEOREM 1.4. Let $T=U|T|$ be the polar decomposition of $T$ in $\mathcal{L}(\mathbf{H})$ and let $D$ be an arbitrary bounded disk containing $\sigma(T) \cup\{0\}$ in $\mathbf{C}$. Then $T-\lambda: W^{2}(D, \mathbf{H}) \rightarrow$ $W^{2}(D, \mathbf{H})$ is one-to-one if and only if $\tilde{T}-\lambda: W^{2}(D, \mathbf{H}) \rightarrow W^{2}(D, \mathbf{H})$ is one-to-one.

Proof. Assume $T-\lambda$ is one-to-one. If $f \in W^{2}(D, \mathbf{H})$ is such that $(\tilde{T}-\lambda) f=0$, then $(T-\lambda) U|T|^{\frac{1}{2}} f=0$. By the hypothesis, $U|T|^{\frac{1}{2}}=0$. Hence $\tilde{T} f=0$. Thus $\lambda f=0$; i.e., $\lambda \bar{\partial}^{i} f=0$ for $i=0,1,2$. By applications of [9, Proposition 3.2] with $T=0$, we get

$$
\|(I-P) f\|_{2, D} \leq C_{D}\left(\|-\lambda \bar{\partial} f\|_{2, D}+\left\|-\lambda \bar{\partial}^{2} f\right\|_{2, D}\right),
$$

where $P$ denotes the orthogonal projection of $L^{2}(D, \mathbf{H})$ onto the Bergman space $A^{2}(D, \mathbf{H})$. From (1) we have $f=P f$. Hence $\lambda f=\lambda P f=0$. From [3, Corollary 10.7], there exists a constant $c>0$ such that

$$
c\|P f\|_{2, D} \leq\|\lambda P f\|_{2, D} .
$$

Hence $f=P f=0$.

Conversely, if $\tilde{T}-\lambda$ is one-to-one, we can prove the required result by the same argument.

The following corollary shows that, for every $p$-hyponormal operator $T$, the equality $\operatorname{supp}((T-\lambda) f)=\operatorname{supp}(f)$ holds for every $f \in W^{2}(D, \mathbf{H})$.

COROLlary 1.5. If $T$ is p-hyponormal, then the operator $T-\lambda: W^{2}(D, \mathbf{H}) \rightarrow$ $W^{2}(D, \mathbf{H})$ is one-to-one.

Proof. Since $\tilde{\tilde{T}}$ is hyponormal by [1], it is known from [9] that $\tilde{\tilde{T}}-\lambda$ is one-to-one. By two applications of Theorem 1.4 we conclude that $T-\lambda$ is one-to-one.

Corollary 1.6. If an operator $T \in \mathcal{L}(\mathbf{H})$ satisfies $T=S+N$, where $S$ is $p$ hyponormal, $S$ and $N$ commute, and $N^{m}=0$, then $T-\lambda$ is one-to-one on $W^{2}(D, \mathbf{H})$.

Proof. Let $f \in W^{2}(D, \mathbf{H})$ be such that $(T-\lambda) f=0$. Then

$$
(S-\lambda) f=-N f .
$$

Hence $(S-\lambda) N^{j-1} f=-N^{j} f$ for $j=1,2, \ldots, m$. We prove that $N^{j} f=0$ for $j=$ $0,1, \ldots, m-1$ by induction. Since $N^{m}=0$,

$$
(S-\lambda) N^{m-1} f=-N^{m} f=0 .
$$

Since $S-\lambda$ is one-to-one from Corollary $1.5, N^{m-1} f=0$. Assume it is true when $j=k$, i.e., $N^{k} f=0$. From (2), we get

$$
(S-\lambda) N^{k-1} f=-N^{k} f=0 .
$$

Since $S-\lambda$ is one-to-one from Corollary $1.5, N^{k-1} f=0$. By induction, we have $f=0$. Hence $T-\lambda$ is one-to-one. 
The following theorem shows that if $\lim _{n \rightarrow \infty}\left\|(T-\lambda) f_{n}\right\|_{W^{m}}=0$, then we cannot obtain by the same method more than $\lim _{n \rightarrow \infty}\left\|f_{n}\right\|_{W^{m-2}}=0$ for $m \geq 2$.

THEOREM 1.7. Let $T=U|T|$ be the polar decomposition of $T$ in $\mathcal{L}(\mathbf{H})$ and let $D$ be an arbitrary bounded disk containing $\sigma(T) \cup\{0\}$ in $\mathbf{C}$. Assume that $\tilde{T}-\lambda: W^{m}(D, \mathbf{H}) \rightarrow$ $W^{m}(D, \mathbf{H})$ is bounded below. If $f_{n}$ is a sequence in $W^{m}(D, \mathbf{H})$ such that we have $\lim _{n \rightarrow \infty}\left\|(T-\lambda) f_{n}\right\|_{W^{m}}=0$, then $\lim _{n \rightarrow \infty}\left\|f_{n}\right\|_{W^{m-2}}=0$ for $m \geq 2$.

Proof. If $f_{n}$ is a sequence in $W^{m}(D, \mathbf{H})$ such that $\lim _{n \rightarrow \infty}\left\|(T-\lambda) f_{n}\right\|_{W^{m}}=0$, then by the definition of the norm in $W^{m}(D, \mathbf{H})$ we have

$$
\lim _{n \rightarrow \infty}\left\|(T-\lambda) \bar{\partial}^{i} f_{n}\right\|_{2, D}=0
$$

for $i=0,1, \ldots, m$. Since $|T|^{1 / 2} T=\tilde{T}|T|^{1 / 2}$, we get

$$
\lim _{n \rightarrow \infty}\left\|(\tilde{T}-\lambda)|T|^{\frac{1}{2}} \bar{\partial}^{i} f_{n}\right\|_{2, D}=0
$$

for $i=0,1, \ldots, m$. Since $\tilde{T}-\lambda$ is bounded below, we have

$$
\lim _{n \rightarrow \infty}\left\||T|^{\frac{1}{2}} \bar{\partial}^{i} f_{n}\right\|_{2, D}=0
$$

for $i=0,1, \ldots, m$. Since $T=U|T|$, we get

$$
\lim _{n \rightarrow \infty}\left\|T \bar{\partial}^{i} f_{n}\right\|_{2, D}=0
$$

for $i=0,1, \ldots, m$. Hence by (3) and (4) we obtain

$$
\lim _{n \rightarrow \infty}\left\|\lambda \bar{\partial}^{i} f_{n}\right\|_{2, D}=0
$$

for $i=0,1, \ldots, m$. By an application of [7, Proposition 2.2] with $T=0$,

$$
\lim _{n \rightarrow \infty}\left\|(I-P) \bar{\partial}^{i} f_{n}\right\|_{2, D}=0
$$

for $i=0,1, \ldots, m-2$, where $P$ denotes the orthogonal projection of $L^{2}(D, \mathbf{H})$ onto the Bergman space $A^{2}(D, \mathbf{H})=L^{2}(D, \mathbf{H}) \cap \mathcal{O}(U, \mathbf{H})$. Then (5) and (6) imply that

$$
\lim _{n \rightarrow \infty}\left\|\lambda P \bar{\partial}^{i} f_{n}\right\|_{2, D}=0
$$

for $i=0,1, \ldots, m-2$. Since $\lambda P \bar{\partial}^{i} f_{n}$ is bounded below, by [3, Corollary 10.7], we get

$$
\lim _{n \rightarrow \infty}\left\|P \bar{\partial}^{i} f_{n}\right\|_{2, D}=0
$$

for $i=0,1, \ldots, m-2$. By (6) and (7) we conclude that $\lim _{n \rightarrow \infty}\left\|f_{n}\right\|_{W^{m-2}}=0$.

Next we show that Aluthge transforms preserve the finite ascent except for $\lambda=0$.

THEOREM 1.8. For arbitrary $\lambda \in \mathbf{C} \backslash\{0\}, \operatorname{ker}(T-\lambda)^{n}=\operatorname{ker}(T-\lambda)^{n+1}$ if and only if $\operatorname{ker}(\tilde{T}-\lambda)^{n}=\operatorname{ker}(\tilde{T}-\lambda)^{n+1}$, for some $n \in \mathbf{N}$.

Proof. Assume that for all $\lambda \in \mathbf{C} \backslash\{0\}$, there is an $n \in \mathbf{N}$ such that $\operatorname{ker}(T-\lambda)^{n}=$ $\operatorname{ker}(T-\lambda)^{n+1}$. Since $\operatorname{ker}(\tilde{T}-\lambda)^{n} \subset \operatorname{ker}(\tilde{T}-\lambda)^{n+1}$, it suffices to show that 
$\operatorname{ker}(\tilde{T}-\lambda)^{n} \supset \operatorname{ker}(\tilde{T}-\lambda)^{n+1}$. Let $x \in \operatorname{ker}(\tilde{T}-\lambda)^{n+1}$. Since $T\left(U|T|^{\frac{1}{2}}\right)=\left(\left.U\right|^{\frac{1}{2}}\right) \tilde{T}$,

$$
(T-\lambda)^{n+1} U|T|^{\frac{1}{2}} x=U|T|^{\frac{1}{2}}(\tilde{T}-\lambda)^{n+1} x=0 .
$$

Therefore, $U|T|^{\frac{1}{2}} x \in \operatorname{ker}(T-\lambda)^{n+1}=\operatorname{ker}(T-\lambda)^{n}$. Since

$$
U|T|^{\frac{1}{2}}(\tilde{T}-\lambda)^{n} x=(T-\lambda)^{n} U|T|^{\frac{1}{2}} x=0,
$$

$\tilde{T}(\tilde{T}-\lambda)^{n} x=0$. We obtain $\lambda(\tilde{T}-\lambda)^{n} x=0$. Since $\lambda \neq 0,(\tilde{T}-\lambda)^{n} x=0$.

The proof of the converse implication is similar.

THEOREM 1.9. Let $T \in \mathcal{L}(\mathbf{H})$ have polar decomposition $U|T|$. Then for all nonzero $\lambda \in \mathbf{C}, \operatorname{ran}(T-\lambda)$ is closed if and only if $\operatorname{ran}(\tilde{T}-\lambda)$ is closed.

Proof. Assume that $\operatorname{ran}(\tilde{T}-\lambda)$ is closed, for all nonzero $\lambda \in \mathbf{C}$. If $y \in \overline{\operatorname{ran}(T-\lambda)}$, for all nonzero $\lambda \in \mathbf{C}$, then there exists a sequence $\left\{x_{n}\right\}$ in $\mathbf{H}$ such that

$$
\lim _{n \rightarrow \infty}(T-\lambda) x_{n}=y \text {. }
$$

Since $|T|^{\frac{1}{2}} T=\tilde{T}|T|^{\frac{1}{2}}$, we have

$$
\lim _{n \rightarrow \infty}(\tilde{T}-\lambda)|T|^{\frac{1}{2}} x_{n}=\lim _{n \rightarrow \infty}|T|^{\frac{1}{2}}(T-\lambda) x_{n}=|T|^{\frac{1}{2}} y .
$$

Since $\operatorname{ran}(\tilde{T}-\lambda)$ is closed, for all nonzero $\lambda \in \mathbf{C}$, there exists a $z \in \mathbf{H}$ such that

$$
\lim _{n \rightarrow \infty}(\tilde{T}-\lambda)|T|^{\frac{1}{2}} x_{n}=(\tilde{T}-\lambda) z
$$

Since the limit is unique, $(\tilde{T}-\lambda) z=|T|^{\frac{1}{2}} y$. Thus $\tilde{T} z=|T|^{\frac{1}{2}} y+\lambda z$. Set $w=\left.U\right|^{\frac{1}{2}} z-y$. Then

$$
|T|^{\frac{1}{2}} w=\tilde{T} z-|T|^{\frac{1}{2}} y=\lambda z
$$

Hence we get

$$
\begin{aligned}
(T-\lambda) w & =U|T|^{\frac{1}{2}}\left(|T|^{\frac{1}{2}} w\right)-\lambda w \\
& =U|T|^{\frac{1}{2}}(\lambda z)-\lambda\left(U|T|^{\frac{1}{2}} z-y\right) \\
& =\lambda y .
\end{aligned}
$$

Since $\lambda$ is nonzero,

$$
(T-\lambda)\left(\frac{w}{\lambda}\right)=y .
$$

Hence $y \in \operatorname{ran}(T-\lambda)$. Thus $\operatorname{ran}(T-\lambda)$ is closed, for all nonzero $\lambda \in \mathbf{C}$.

The proof of the converse is similar.

Corollary 1.10. For all nonzero $\lambda \in \mathbf{C}, T-\lambda$ is bounded below if and only if $\tilde{T}-\lambda$ is.

Proof. Let $T=U|T|$ be the polar decomposition of $T$. If $T-\lambda$ is bounded below for all nonzero $\lambda \in \mathbf{C}$, then it is one-to-one and has closed range. From Theorem 1.9, 
it suffices to show that $\tilde{T}-\lambda$ is one-to-one. If $(\tilde{T}-\lambda) x=0$, then $(T-\lambda) U|T|^{\frac{1}{2}} x=0$. Hence $U|T|^{\frac{1}{2}} x=0$, i.e., $\tilde{T} x=0$. Since $\lambda \neq 0, x=0$.

The proof of the converse is similar.

The following theorem shows that the Aluthge transform preserves the finite descent except for $\lambda=0$.

THEOREM 1.11. For all nonzero $\lambda \in \mathbf{C}, \operatorname{ran}(T-\lambda)^{n}=\operatorname{ran}(T-\lambda)^{n+1}$ if and only if $\operatorname{ran}(\tilde{T}-\lambda)^{n}=\operatorname{ran}(\tilde{T}-\lambda)^{n+1}$ for some $n \in \mathbf{N}$.

Proof. Assume that $\operatorname{ran}(T-\lambda)^{n}=\operatorname{ran}(T-\lambda)^{n+1}$ for some $n \in \mathbf{N}$ and for all nonzero $\lambda \in \mathbf{C}$. Since $\operatorname{ran}(\tilde{T}-\lambda)^{n} \supset \operatorname{ran}(\tilde{T}-\lambda)^{n+1}$, it suffices to show that $\operatorname{ran}(\tilde{T}-\lambda)^{n} \subset \operatorname{ran}(\tilde{T}-\lambda)^{n+1}$. If $y \in \operatorname{ran}(\tilde{T}-\lambda)^{n}$, there exists an $x \in \mathbf{H}$ such that $y=\operatorname{ran}(\tilde{T}-\lambda)^{n} x$. Since $U|T|^{\frac{1}{2}} \tilde{T}=T U|T|^{\frac{1}{2}}$,

$$
U|T|^{\frac{1}{2}} y=(T-\lambda)^{n} U|T|^{\frac{1}{2}} x .
$$

Since $U|T|^{\frac{1}{2}} y \in \operatorname{ran}(T-\lambda)^{n}=\operatorname{ran}(T-\lambda)^{n+1}$, there exists a $z \in \mathbf{H}$ such that $\tilde{T} y=$ $|T|^{\frac{1}{2}}(T-\lambda)^{n+1} z=(\tilde{T}-\lambda)^{n+1}|T|^{\frac{1}{2}} z$. Hence $\tilde{T} y \in \operatorname{ran}(\tilde{T}-\lambda)^{n+1}$ and so there exists an $s \in \mathbf{H}$ such that $\tilde{T} y=(\tilde{T}-\lambda)^{n+1} s$. Set $w=(\tilde{T}-2 \lambda) s-(\tilde{T}-\lambda)^{2} s$. Then

$$
(\tilde{T}-\lambda)^{n+1} w=-\lambda^{2} y .
$$

Since $\lambda \neq 0$,

$$
(\tilde{T}-\lambda)^{n+1}\left(-\frac{w}{\lambda^{2}}\right)=y
$$

Hence $y \in \operatorname{ran}(\tilde{T}-\lambda)^{n+1}$.

The proof of the converse is similar.

Suppose that $T \in \mathcal{L}(\mathbf{H})$ has the single valued extension property. The operator $T$ is said to satisfy Dunford's property $(C)$ if the linear submanifold

$$
H_{T}(F):=\left\{x \in \mathbf{H}: \sigma_{T}(x) \subseteq F\right\}
$$

is closed, for each closed subset $F$ of $\mathbf{C}$, where $\sigma_{T}(x):=\mathbf{C} \backslash \rho_{T}(x)$.

The following theorem shows that Aluthge transforms preserve Dunford's property (C) in some cases.

Recall that an operator $X \in \mathcal{L}(\mathbf{H}, \mathbf{K})$ is called a quasiaffinity if it has trivial kernel and dense range. An operator $A \in \mathcal{L}(\mathbf{H})$ is said to be a quasiaffine transform of an operator $T \in \mathcal{L}(\mathbf{K})$ if there is a quasiaffinity $X \in \mathcal{L}(\mathbf{H}, \mathbf{K})$ such that $X A=T X$. Furthermore, operators $A$ and $T$ are said to be quasisimilar if there are quasiaffinities $X$ and $Y$ such that $X A=T X$ and $A Y=Y T$.

THEOREM 1.12. If $T$, with polar decomposition $U|T|$ is a quasiaffinity in $\mathcal{L}(\mathbf{H})$, then $T$ satisfies Dunford's property $(C)$ if and only if $\tilde{T}$ does.

Proof. Assume that $T$ satisfies Dunford's property $(C)$. Consider

$$
H_{\tilde{T}}(F):=\left\{x \in \mathbf{H}: \sigma_{\tilde{T}}(x) \subseteq F\right\},
$$


for every closed subset $F$ of $\mathbf{C}$. Since $\tilde{T}$ has the single valued extension property from Theorem 1.1, it suffices to show that $H_{\tilde{T}}(F)$ is closed. If $x \in \overline{H_{\tilde{T}}(F)}$, then there exist a sequence $\left\{x_{n}\right\}$ in $H_{\tilde{T}}(F)$ such that $x_{n} \rightarrow x$. Since $x_{n} \in H_{\tilde{T}}(F), \sigma_{\tilde{T}}\left(x_{n}\right) \subseteq F$. For any $\lambda \in F^{c}$ we have $\lambda \in \rho_{\tilde{T}}\left(x_{n}\right)$. Hence $(\tilde{T}-\lambda) x_{n}(\lambda) \equiv x_{n}$, where $\lambda \rightarrow x_{n}(\lambda)$ is the analytic function defined on $\rho_{\tilde{T}}\left(x_{n}\right)$. Since $U|T|^{\frac{1}{2}} \tilde{T}=T U|T|^{\frac{1}{2}}$,

$$
(T-\lambda) U|T|^{\frac{1}{2}} x_{n}(\lambda) \equiv U|T|^{\frac{1}{2}} x_{n} .
$$

Hence $\lambda \in \rho_{T}\left(U|T|^{\frac{1}{2}} x_{n}\right)$. Thus $\sigma_{T}\left(U|T|^{\frac{1}{2}} x_{n}\right) \subseteq F$. Therefore,

$$
U|T|^{\frac{1}{2}} x_{n} \in H_{T}(F) .
$$

Since $H_{T}(F)$ is closed by hypothesis, $U|T|^{\frac{1}{2}} x \in H_{T}(F)$. For any $\lambda \in F^{c}$, we have

$$
(T-\lambda) U|T|^{\frac{1}{2}} x(\lambda) \equiv U|T|^{\frac{1}{2}} x .
$$

Since $U|T|^{\frac{1}{2}} \tilde{T}=T U|T|^{\frac{1}{2}}$, we have

$$
U|T|^{\frac{1}{2}}(\tilde{T}-\lambda) x(\lambda) \equiv U|T|^{\frac{1}{2}} x .
$$

Since $T$ is a quasiaffinity, we get

$$
(\tilde{T}-\lambda) x(\lambda) \equiv x
$$

Thus $\lambda \in \rho_{\tilde{T}}(x)$. Hence $\sigma_{\tilde{T}}(x) \subseteq F$.

The proof of the converse implication is similar.

An operator $T \in \mathcal{L}(\mathbf{H})$ is called decomposable if for every finite open covering $\left\{G_{1}, \ldots, G_{n}\right\}$ of $\mathbf{C}$ there exists a system $\left\{Y_{1}, \ldots, Y_{n}\right\}$ of spectral maximal subspaces of $T$ such that $\mathbf{H}=Y_{1}+\cdots+Y_{n}$ and $\sigma\left(\left.T\right|_{Y_{i}}\right) \subset G_{i}$ for every $1 \leq i \leq n$. As one of the generalized concepts of decomposability, we define the following; an operator $T \in \mathcal{L}(\mathbf{H})$ is quasidecomposable if $T$ has Dunford's property $(C)$ and satisfies the condition that for every finite open covering $\left\{G_{1}, \ldots, G_{n}\right\}$ of $\mathbf{C}$ there corresponds a system $\left\{Y_{1}, \ldots, Y_{n}\right\}$ of $T$-invariant subspaces such that $\mathbf{H}=\vee_{i=1}^{n} Y_{i}$ and $\sigma\left(\left.T\right|_{Y_{i}}\right) \subset G_{i}$ for every $1 \leq i \leq n$. As an application of Theorem 1.7 we have the following corollary.

COROllary 1.13. Let $T$ with polar decomposition $U|T|$ be a quasiaffinity in $\mathcal{L}(\mathbf{H})$. If $\tilde{T}$ is decomposable, then $T$ is quasidecomposable.

Proof. If $\tilde{T}$ is decomposable, it has Dunford's property $(C)$ from [8]. Then $T$ has Dunford's property $(C)$, by Theorem 1.12. Since $T U|T|^{\frac{1}{2}}=U|T|^{\frac{1}{2}} \tilde{T}$, Corollary 1.3 implies that

$$
U|T|^{\frac{1}{2}} H_{\tilde{T}}(F) \subset H_{T}(F),
$$

for each closed $F$. Let $\left\{G_{1}, \ldots, G_{n}\right\}$ be an open cover of $\mathbf{C}$. Then

$$
\mathbf{H}=H_{\tilde{T}}\left(\bar{G}_{1}\right)+\cdots+H_{\tilde{T}}\left(\bar{G}_{n}\right)
$$

Since $\overline{U|T|^{\frac{1}{2}} \mathbf{H}}=\mathbf{H}$, we have

$$
U|T|^{\frac{1}{2}} H_{\tilde{T}}\left(\bar{G}_{1}\right)+\cdots+U|T|^{\frac{1}{2}} H_{\tilde{T}}\left(\bar{G}_{n}\right) \subset H_{T}\left(\bar{G}_{1}\right)+\cdots+H_{T}\left(\bar{G}_{n}\right) .
$$


Hence

$$
\begin{aligned}
\mathbf{H}=\overline{U|T|^{\frac{1}{2}} \mathbf{H}} & =\overline{U|T|^{\frac{1}{2}}\left[H_{\tilde{T}}\left(\bar{G}_{1}\right)+\cdots+H_{\tilde{T}}\left(\bar{G}_{n}\right)\right]} \\
& \subset \overline{H_{T}\left(\bar{G}_{1}\right)+\cdots+H_{T}\left(\bar{G}_{n}\right)} .
\end{aligned}
$$

Thus

$$
\mathbf{H}=\vee_{i=1}^{n} H_{T}\left(\bar{G}_{i}\right)
$$

Since $T$ has Dunford's property $(C)$, by [2, Proposition 3.8]

$$
\sigma\left(\left.T\right|_{H_{T}\left(\bar{G}_{i}\right)}\right) \subset \bar{G}_{i},
$$

for each $i$, so that $T$ is quasidecomposable.

An operator $T \in \mathcal{L}(\mathbf{H})$ is said to satisfy the property $(\beta)$ if for every open subset $G$ of $\mathbf{C}$ and every sequence $f_{n}: G \longrightarrow \mathbf{H}$ of $\mathbf{H}$-valued analytic functions such that $(T-\lambda) f_{n}(\lambda)$ converges uniformly to 0 in norm on compact subsets of $G, f_{n}(\lambda)$ converges uniformly to 0 in norm on compact subsets of $G$.

The following theorem shows that Aluthge transforms preserve the property $(\beta)$.

THEOREM 1.14. An operator $T$ with polar decomposition $U|T|$ satisfies the property $(\beta)$ if and only if an operator $\tilde{T}$ does.

Proof. Assume $T$ satisfies the property $(\beta)$. Let $f_{n} \in \mathcal{O}(V, \mathbf{H})$ be such that $(\tilde{T}-\lambda) f_{n}(\lambda)$ converges uniformly to 0 on compact subsets $G$ of $V$. Since $T\left(\left.U\right|^{\frac{1}{2}}\right)=$ $\left(U|T|^{\frac{1}{2}}\right) \tilde{T},(T-\lambda) U|T|^{\frac{1}{2}} f_{n}(\lambda)$ converges uniformly to 0 for all $\lambda \in G$. Since $T$ satisfies the property $(\beta), U|T|^{\frac{1}{2}} f_{n}(\lambda)$ converges uniformly to 0 for all $\lambda \in G$. Since $\tilde{T}=$ $|T|^{\frac{1}{2}} U|T|^{\frac{1}{2}}, \tilde{T} f_{n}(\lambda)$ converges uniformly to 0 for all $\lambda \in G$. Hence $\lambda f_{n}(\lambda)$ converges uniformly to 0 for all $\lambda \in G$. Since 0 is hyponormal and hyponormal operators satisfy the property $(\beta), f_{n}(\lambda)$ converges uniformly to 0 for all $\lambda \in G$. Hence $\tilde{T}$ satisfies the property $(\beta)$.

The proof of the converse is similar.

COROLlaRY 1.15. If $\tilde{T}$ is algebraic (i.e., $p(\tilde{T})=0$ for some nonzero polynomial $p$ ), then $T=U|T|$ (polar decomposition) satisfies the property $(\beta)$.

Proof. If $\tilde{T}$ is algebraic, then it satisfies the property $(\beta)$ by [6]. Hence, by Theorem $1.14, T$ satisfies the property $(\beta)$.

As an application of Theorem 1.14, we have the following corollary.

COROLlary 1.16. If $T$ is p-hyponormal, then it satisfies the property $(\beta)$.

Proof. Since $\tilde{\tilde{T}}$ is hyponormal by [1], it satisfies the property $(\beta)$. Hence from two applications of Theorem 1.14, $T$ satisfies the property $(\beta)$.

COROLlary 1.17. Suppose that $T$ is p-hyponormal and $S$ satisfies the property $(\beta)$. If $S$ and $T$ are quasisimilar, then $S$ satisfies Weyl's theorem (i.e., $\sigma(T)-\omega(T)=\pi_{00}(T)$, where $\pi_{00}(T)$ denotes the set of all eigenvalues of finite multiplicity of $T$ and $\omega(T)$ denotes the Weyl spectrum of $T$ ). 
Proof. Since $T$ satisfies the property $(\beta)$, by Corollary 1.16, [10] implies that $S$ satisfies Weyl's theorem.

\section{REFERENCES}

1. A. Aluthge, On $p$-hyponormal operators for $0<p<1$, Int. Eq. Op. Th. 13 (1990), 307315 .

2. I. Colojoară and C. Foiaş, Theory of generalized spectral operators (Gordon and Breach, New York, 1968).

3. J. B. Conway, Subnormal operators (Pitman, London, 1981).

4. J. Eschmeier and M. Putinar, Bishop's condition $(\beta)$ and rich extensions of linear operators, Indiana Univ. Math. J. 37 (1988), 325-348.

5. I. Jung, E. Ko and C. Pearcy, Aluthge transforms of operators, Int. Eq. Op. Th. 38 (2000), 437-448.

6. E. Ko, Algebraic and triangular $n$-hyponormal operators, Proc. Amer. Math. Soc. 123 (1995), 3473-3481.

7. R. Lange and S. Wang, New approaches in spectral decomposition, Contemporary Math. No. 128 ( Amer. Math. Soc., 1992).

8. M. Martin and M. Putinar, Lectures on hyponormal operators, Op. Th. Adv. Appl. 39 (Birkhäuser-Verlag, 1989). 395.

9. M. Putinar, Hyponormal operators are subscalar, J. Operator Theory 12 (1984), 385

10. M. Putinar, Quasisimilarity of tuples with Bishop's property ( $\beta$ ), Int. Eq. Op. Th. 15 (1992), 1047-1052. 